\title{
Split equality problem and multiple-sets split equality problem for quasi-nonexpansive multi-valued mappings
}

\author{
Yujing $\mathrm{Wu}^{1}$, Rudong Chen ${ }^{2 *}$ and Luo Yi Shi ${ }^{2}$
}

\section{"Correspondence:}

chenrd@tjpu.edu.cn

${ }^{2}$ Department of Mathematics,

Tianjin Polytechnic University,

Tianjin, 300387, P.R. China

Full list of author information is

available at the end of the article

\begin{abstract}
The multiple-sets split equality problem (MSSEP) requires finding a point $x \in \bigcap_{i=1}^{N} C_{i}$, $y \in \bigcap_{j=1}^{M} Q_{j}$, such that $A x=B y$, where $N$ and $M$ are positive integers, $\left\{C_{1}, C_{2}, \ldots, C_{N}\right\}$ and $\left\{Q_{1}, Q_{2}, \ldots, Q_{M}\right\}$ are closed convex subsets of Hilbert spaces $H_{1}, H_{2}$, respectively, and $A: H_{1} \rightarrow H_{3}, B: H_{2} \rightarrow H_{3}$ are two bounded linear operators. When $N=M=1$, the MSSEP is called the split equality problem (SEP). If let $B=I$, then the MSSEP and SEP reduce to the well-known multiple-sets split feasibility problem (MSSFP) and split feasibility problem (SFP), respectively. Recently, some authors proposed many algorithms to solve the SEP and MSSEP. However, to implement these algorithms, one has to find the projection on the closed convex sets, which is not possible except in simple cases. One of the purposes of this paper is to study the SEP and MSSEP for a family of quasi-nonexpansive multi-valued mappings in the framework of infinite-dimensional Hilbert spaces, and propose an algorithm to solve the SEP and MSSEP without the need to compute the projection on the closed convex sets.
\end{abstract}

Keywords: split equality problem; iterative algorithms; converge strongly

\section{Introduction and preliminaries}

Throughout this paper, we assume that $H$ is a real Hilbert space, $C$ is a subset of $H$. Denote by $C B(H)$ the collection of all nonempty closed and bounded subsets of $H$ and by $\operatorname{Fix}(T)$ the set of the fixed points of a mapping $T$. The Hausdorff metric $\tilde{H}$ on $C B(H)$ is defined by

$$
\tilde{H}(C, D):=\max \left\{\sup _{x \in C} d(x, D), \sup _{y \in D} d(y, C)\right\}, \quad \forall C, D \in C B(H),
$$

where $d(x, K):=\inf _{y \in K} d(x, y)$.

Definition 1.1 Let $R: H \rightarrow C B(H)$ be a multi-valued mapping. An element $p \in H$ is said to be a fixed point of $R$, if $p \in R p$. The set of fixed points of $R$ will be denoted by $\operatorname{Fix}(R)$. $R$ is said to be

(i) nonexpansive, if $\tilde{H}(R x, R y) \leq\|x-y\|, \forall x, y \in H$;

(ii) quasi-nonexpansive, if $\operatorname{Fix}(R) \neq \emptyset$ and $\tilde{H}(R x, R y) \leq\|x-y\|, \forall x \in H, y \in \operatorname{Fix}(R)$.

Let $\left\{C_{1}, C_{2}, \ldots, C_{N}\right\}$ and $\left\{Q_{1}, Q_{2}, \ldots, Q_{M}\right\}$ be nonempty closed convex subsets of real Hilbert spaces $H_{1}$ and $H_{2}$, respectively, and let $A: H_{1} \rightarrow H_{2}$ be a bounded linear operator.

\section{Springer}

@2014 Wu et al.: licensee Springer. This is an Open Access article distributed under the terms of the Creative Commons Attribution License (http://creativecommons.org/licenses/by/2.0), which permits unrestricted use, distribution, and reproduction in any medium, provided the original work is properly cited. 
Recall that the multiple-sets split feasibility problem (MSSFP) is to find a point $x$ satisfying the property:

$$
x \in \bigcap_{i=1}^{N} C_{i}, \quad A x \in \bigcap_{j=1}^{M} Q_{j},
$$

if such a point exists. If $N=M=1$, then the MSSFP reduce to the well-known split feasibility problem (SFP).

The SFP and MSSFP was first introduced by Censor and Elfving [1], and Censor $e$ a al. [2], respectively, which attracted many authors' attention due to the applications in signal processing [1] and intensity-modulated radiation therapy [2]. Various algorithms have been invented to solve it (see [1-8], etc.).

Recently, Moudafi [9] proposed a new split equality problem (SEP): Let $H_{1}, H_{2}, H_{3}$ be real Hilbert spaces, $C \subseteq H_{1}, Q \subseteq H_{2}$ be two nonempty closed convex sets, and let $A: H_{1} \rightarrow H_{3}$, $B: H_{2} \rightarrow H_{3}$ be two bounded linear operators. Find $x \in C, y \in Q$ satisfying

$$
A x=B y .
$$

When $B=I$, SEP reduces to the well-known SFP.

Naturally, we propose the following multiple-sets split equality problem (MSSEP) requiring to find a point $x \in \bigcap_{i=1}^{N} C_{i}, y \in \bigcap_{j=1}^{M} Q_{j}$, such that

$$
A x=B y,
$$

where $N$ and $M$ are positive integers, $\left\{C_{1}, C_{2}, \ldots, C_{N}\right\}$ and $\left\{Q_{1}, Q_{2}, \ldots, Q_{M}\right\}$ are closed convex subsets of Hilbert spaces $H_{1}, H_{2}$, respectively, and $A: H_{1} \rightarrow H_{3}, B: H_{2} \rightarrow H_{3}$ are two bounded linear operators.

In the paper [9], Moudafi give the alternating CQ-algorithm and relaxed alternating CQ-algorithm iterative algorithm for solving the split equality problem.

Let $S=C \times Q$ in $H=H_{1} \times H_{2}$, define $G: H \rightarrow H_{3}$ by $G=[A,-B]$, then $G^{*} G: H \rightarrow H$ has the matrix form

$$
G^{*} G=\left[\begin{array}{cc}
A^{*} A & -A^{*} B \\
-B^{*} A & B^{*} B
\end{array}\right] .
$$

The original problem can now be reformulated as finding $w=(x, y) \in S$ with $G w=0$, or, more generally, minimizing the function $\|G w\|$ over $w \in S$. Therefore solving SEP (1.1) is equivalent to solving the following minimization problem:

$$
\min _{w \in S} f(w)=\frac{1}{2}\|G w\|^{2} .
$$

In the paper [10], we use the well-known Tychonov regularization to get some algorithms that converge strongly to the minimum-norm solution of the SEP.

Note that to implement these algorithms, one has to find the projection on the closed convex sets, which is not possible except in simple cases. 
The purpose of this paper is to introduce and study the following split equality problem for quasi-nonexpansive multi-valued mappings in infinitely dimensional Hilbert spaces, i.e., to find $w=(x, y) \in C$ such that

$$
A x=B y,
$$

where $H_{1}, H_{2}, H_{3}$ are real Hilbert spaces, $A: H_{1} \rightarrow H_{3}, B: H_{2} \rightarrow H_{3}$ are two bounded linear operators, $R_{i}: H_{i} \rightarrow C B\left(H_{i}\right), i=1,2$ are two quasi-nonexpansive multi-valued mappings, $C=\operatorname{Fix}\left(R_{1}\right), Q=\operatorname{Fix}\left(R_{2}\right)$. In the rest of this paper, we still use $\Gamma$ to denote the set of solutions of SEP (1.3), and assume consistency of SEP so that $\Gamma$ is closed, convex, and nonempty, i.e., $\Gamma=\left\{(x, y) \in H_{1} \times H_{2}, A x=B y, x \in C, y \in Q\right\} \neq \emptyset$. The multiple-sets split equality problem (MSSEP) for a family quasi-nonexpansive multi-valued mappings in infinitely dimensional Hilbert spaces, i.e., to find $w=(x, y) \in C$ such that

$$
A x=B y,
$$

where $R_{i}^{j}: H_{i} \rightarrow C B\left(H_{i}\right), i=1,2, j=1,2, \ldots, N$ is a family of quasi-nonexpansive multivalued mappings, $C=\bigcap_{j=1}^{N} \operatorname{Fix}\left(R_{1}^{j}\right), Q=\bigcap_{j=1}^{N} \operatorname{Fix}\left(R_{2}^{j}\right)$. In the rest of this paper, we use $\bar{\Gamma}$ to denote the set of solutions of MSSEP (1.4), and assume consistency of MSSEP so that $\bar{\Gamma}$ is closed, convex, and nonempty, i.e., $\bar{\Gamma}=\left\{(x, y) \in H_{1} \times H_{2}, A x=B y, x \in C, y \in Q\right\} \neq \emptyset$.

In this paper, we study the SEP and MSSEP for a family of quasi-nonexpansive multivalued mappings in the framework of infinite-dimensional Hilbert spaces, and propose an algorithm to solve the SEP and MSSEP not requiring to compute the projection on the closed convex sets.

We now collect some definitions and elementary facts which will be used in the proofs of our main results.

Definition 1.2 Let $H$ be a Banach space.

(1) A multi-valued mapping $R: H \rightarrow C B(H)$ is said to be demi-closed at the origin if, for any sequence $\left\{x_{n}\right\} \subseteq H$ with $x_{n}$ converges weakly to $x$ and $d\left(x_{n}, R x_{n}\right) \rightarrow 0$, we have $x \in R x$.

(2) A multi-valued mapping $R: H \rightarrow C B(H)$ is said to be semi-compact if, for any bounded sequence $\left\{x_{n}\right\} \subseteq H$ with $d\left(x_{n}, R x_{n}\right) \rightarrow 0$, there exists a subsequence $\left\{x_{n_{k}}\right\}$ such that $\left\{x_{n_{k}}\right\}$ converges strongly to a point $x \in H$.

Lemma 1.3 $[11,12]$ Let $X$ be a Banach space, $C$ a closed convex subset of $X$, and $T: C \rightarrow C$ a nonexpansive mapping with $\operatorname{Fix}(T) \neq \emptyset$. If $\left\{x_{n}\right\}$ is a sequence in $C$ weakly converging to $x$ and if $\left\{(I-T) x_{n}\right\}$ converges strongly to $y$, then $(I-T) x=y$.

Lemma 1.4 [13] Let $H$ be a Hilbert space and $\left\{w_{n}\right\}$ a sequence in $H$ such that there exists a nonempty set $S \subseteq H$ satisfying the following:

(i) for every $w \in S, \lim _{n \rightarrow \infty}\left\|w_{n}-w\right\|$ exists;

(ii) any weak-cluster point of the sequence $\left\{w_{n}\right\}$ belongs to $S$.

Then there exists $\tilde{w} \in$ s such that $\left\{w_{n}\right\}$ weakly converges to $\tilde{w}$.

Lemma $1.5[10]$ Let $T=I-\gamma G^{*} G$, where $0<\gamma<\lambda=2 / \rho\left(G^{*} G\right)$ with $\rho\left(G^{*} G\right)$ being the spectral radius of the self-adjoint operator $G^{*} G$ on $H, S=C \times Q$. Then we have the following: 
(1) $\|T\| \leq 1$ (i.e., $T$ is nonexpansive) and averaged;

(2) $\operatorname{Fix}(T)=\{(x, y) \in H, A x=B y\}, \operatorname{Fix}\left(P_{S} T\right)=\operatorname{Fix}\left(P_{S}\right) \cap \operatorname{Fix}(T)=\Gamma$.

\section{Iterative algorithm for SEP}

In this section, we establish an iterative algorithm that converges strongly to a solution of SEP (1.3).

Algorithm 2.1 For an arbitrary initial point $w_{0}=\left(x_{0}, y_{0}\right)$, the sequence $\left\{w_{n}=\left(x_{n}, y_{n}\right)\right\}$ is generated by the iteration:

$$
w_{n+1}=\alpha_{n}\left(I-\gamma G^{*} G\right) w_{n}+\left(1-\alpha_{n}\right) v_{n}, \quad v_{n} \in R\left(w_{n}-\gamma G^{*} G w_{n}\right) \text {, }
$$

where $\alpha_{n}>0$ is a sequence in $(0,1)$ and $0<\gamma<\lambda=2 / \rho\left(G^{*} G\right)$ with $\rho\left(G^{*} G\right)$ being the spectral radius of the self-adjoint operator $G^{*} G$ on $H, R: H_{1} \times H_{2} \rightarrow H_{1} \times H_{2}$ by

$$
R=\left[\begin{array}{cc}
R_{1} & 0 \\
0 & R_{2}
\end{array}\right],
$$

and $R_{1}, R_{2}$ are quasi-nonexpansive multi-valued mappings on $H_{1}, H_{2}$, respectively.

To prove its convergence we need the following lemma.

Lemma 2.2 Any sequence $\left\{w_{n}\right\}$ generated by Algorithm (2.1) is Féjer-monotone with respect to $\Gamma$, namely for every $w \in \Gamma$,

$$
\left\|w_{n+1}-w\right\| \leq\left\|w_{n}-w\right\|, \quad \forall n \geq 1,
$$

provided that $\alpha_{n}>0$ is a sequence in $(0,1)$ and $0<\gamma<\lambda=2 / \rho\left(G^{*} G\right)$.

Proof Let $u_{n}=\left(I-\gamma G^{*} G\right) w_{n}$ and taking $w \in \Gamma$, by Lemma 1.5, $w \in \operatorname{Fix}\left(P_{S}\right) \cap \operatorname{Fix}\left(I-\gamma G^{*} G\right)$, $G w=0$ and we have

$$
\begin{aligned}
\left\|w_{n+1}-w\right\|^{2} & =\left\|\alpha_{n} u_{n}+\left(1-\alpha_{n}\right) v_{n}-w\right\|^{2} \\
& \leq \alpha_{n}\left\|u_{n}-w\right\|^{2}+\left(1-\alpha_{n}\right)\left\|v_{n}-w\right\|^{2}-\alpha_{n}\left(1-\alpha_{n}\right)\left\|u_{n}-v_{n}\right\|^{2} \\
& \leq\left(1-\alpha_{n}\right)\left\|u_{n}-w\right\|^{2}+\alpha_{n} \tilde{H}\left(R u_{n}-R w\right)^{2}-\alpha_{n}\left(1-\alpha_{n}\right)\left\|u_{n}-v_{n}\right\|^{2} \\
& \leq\left(1-\alpha_{n}\right)\left\|u_{n}-w\right\|^{2}+\alpha_{n}\left\|u_{n}-w\right\|^{2}-\alpha_{n}\left(1-\alpha_{n}\right)\left\|u_{n}-v_{n}\right\|^{2} \\
& =\left\|u_{n}-w\right\|^{2}-\alpha_{n}\left(1-\alpha_{n}\right)\left\|u_{n}-v_{n}\right\|^{2} .
\end{aligned}
$$

On the other hand, we have

$$
\begin{aligned}
\left\|u_{n}-w\right\|^{2} & =\left\|\left(I-\gamma G^{*} G\right) w_{n}-w\right\|^{2} \\
& =\left\|w_{n}-w\right\|^{2}+\left\|\gamma G^{*} G w_{n}\right\|^{2}-2\left\langle w_{n}-w, \gamma G^{*} G w_{n}\right\rangle \\
& =\left\|w_{n}-w\right\|^{2}+\gamma^{2}\left\langle G w_{n}, G G^{*} G w_{n}\right\rangle-2 \gamma\left\langle G w_{n}-G w, G w_{n}\right\rangle \\
& \leq\left\|w_{n}-w\right\|^{2}+\gamma^{2} \lambda\left\|G w_{n}\right\|^{2}-2 \gamma\left\langle G w_{n}-0, G w_{n}\right\rangle \\
& =\left\|w_{n}-w\right\|^{2}-\gamma(2-\lambda \gamma)\left\|G w_{n}\right\|^{2} .
\end{aligned}
$$


Hence, we have

$$
\left\|w_{n+1}-w\right\|^{2} \leq\left\|w_{n}-w\right\|^{2}-\alpha_{n}\left(1-\alpha_{n}\right)\left\|u_{n}-v_{n}\right\|^{2}-\gamma(2-\lambda \gamma)\left\|G w_{n}\right\|^{2} .
$$

It follows that $\left\|w_{n+1}-w\right\| \leq\left\|w_{n}-w\right\|, \forall w \in \Gamma, n \geq 1$.

Theorem 2.3 If $0<\liminf _{n \rightarrow \infty} \alpha_{n} \leq \limsup _{n \rightarrow \infty} \alpha_{n}<1$ and $R_{1}, R_{2}$ are demi-closed at the origin, then the sequence $\left\{w_{n}\right\}$ generated by Algorithm (2.1) converges weakly to a solution of SEP (1.3). In addition, if $R_{1}, R_{2}$ are semi-compact, then $\left\{w_{n}\right\}$ converges strongly to a solution of SEP (1.3).

Proof For any solution of SEP $w$, according to Lemma 2.2, we see that the sequence $\left\|w_{n}-w\right\|$ is monotonically decreasing and thus converges to some positive real. Since $0<\liminf _{n \rightarrow \infty} \alpha_{n} \leq \limsup _{n \rightarrow \infty} \alpha_{n}<1$ and $0<\gamma<\lambda$, by (2.2), we can obtain

$$
\left\|u_{n}-v_{n}\right\| \rightarrow 0, \quad\left\|G w_{n}\right\| \rightarrow 0, \quad \text { when } n \rightarrow \infty
$$

Since $v_{n} \in R u_{n}$, we can get $d\left(u_{n}, R u_{n}\right) \leq\left\|u_{n}-v_{n}\right\| \rightarrow 0$.

From the Féjer-monotonicity of $\left\{w_{n}\right\}$ it follows that the sequence is bounded. Denoting by $\tilde{w}$ a weak-cluster point of $\left\{w_{n}\right\}$ let $v=0,1,2, \ldots$ be the sequence of indices, such that $w_{n_{v}}$ converges weakly to $\tilde{w}$. Then, by Lemma 1.3 , we obtain $G \tilde{w}=0$, and it follows that $\tilde{w} \in \operatorname{Fix}\left(I-\gamma G^{*} G\right)$.

Since $R_{1}, R_{2}$ are demi-closed at the origin, it is easy to check that $R$ is demi-closed at the origin. Now, by setting $u_{n}=\left(I-\gamma G^{*} G\right) w_{n}$, it follows that $u_{n_{v}}$ converges weakly to $\tilde{w}$. Since $d\left(u_{n}, R u_{n}\right) \rightarrow 0$, and $R$ is demi-closed at the origin, we obtain $\tilde{w} \in \operatorname{Fix} R=C \times Q$, i.e., $P_{S}(\tilde{w})=\tilde{w}$. That is to say, $\tilde{w} \in \operatorname{Fix}\left(P_{S}\right)$.

Hence $\tilde{w} \in \operatorname{Fix}\left(P_{S}\right) \cap \operatorname{Fix}\left(I-\gamma G^{*} G\right)$. By Lemma 1.5, we find that $\tilde{w}$ is a solution of SEP (1.3).

The weak convergence of the whole sequence $\left\{w_{n}\right\}$ holds true since all conditions of the well-known Opial lemma (Lemma 1.4) are fulfilled with $S=\Gamma$.

Moreover, if $R_{1}, R_{2}$ are semi-compact, it is easy to prove that $R$ is semi-compact, and since $d\left(u_{n}, R u_{n}\right) \rightarrow 0$, we get the result that there exists a subsequence of $\left\{u_{n_{i}}\right\} \subseteq\left\{u_{n}\right\}$ such that $u_{n_{i}}$ converges strongly to $w^{*}$. Since $u_{n_{v}}$ converges weakly to $\tilde{w}$, we have $w^{*}=\tilde{w}$ and so $u_{n_{i}}$ converges strongly to $\tilde{w} \in \Gamma$. From the Féjer-monotonicity of $\left\{w_{n}\right\}$ and $\left\|w_{n+1}-u_{n}\right\|=$ $\left(1-\alpha_{n}\right)\left\|u_{n}-v_{n}\right\| \rightarrow 0$, we can find that $\left\|w_{n}-\tilde{w}\right\| \rightarrow 0$, i.e., $\left\{w_{n}\right\}$ converges strongly to a solution of the SEP (1.3).

\section{Iterative algorithm for MSSEP}

In this section, we establish an iterative algorithm that converges strongly to a solution of the following MSSEP (1.4) for a family quasi-nonexpansive multi-valued mappings in infinitely dimensional Hilbert spaces.

Let $C_{j}=\operatorname{Fix} R_{1}^{j}, Q_{j}=\operatorname{Fix} R_{2}^{j}$ and $S_{j}=C_{j} \times Q_{j}, j=1,2, \ldots, N, S=\bigcap_{j=1}^{N} S_{j}$. The original problem can now be reformulated as finding $w=(x, y) \in S$ with $G w=0$, or, more generally, minimizing the function $\|G w\|$ over $w \in S$.

Algorithm 3.1 For an arbitrary initial point $w_{0}=\left(x_{0}, y_{0}\right)$, sequence $\left\{w_{n}=\left(x_{n}, y_{n}\right)\right\}$ is generated by the iteration:

$$
w_{n+1}=\alpha_{n}\left(I-\gamma G^{*} G\right) w_{n}+\left(1-\alpha_{n}\right) v_{n}, \quad v_{n} \in R_{i(n)}\left(w_{n}-\gamma G^{*} G w_{n}\right) \text {, }
$$


where $i(n)=n(\bmod N)+1, \alpha_{n}>0$ is a sequence in $(0,1)$ and $0<\gamma<\lambda=2 / \rho\left(G^{*} G\right), R_{i(n)}$ : $H_{1} \times H_{2} \rightarrow H_{1} \times H_{2}$ by

$$
R_{i(n)}=\left[\begin{array}{cc}
R_{1}^{i(n)} & 0 \\
0 & R_{2}^{i(n)}
\end{array}\right],
$$

and $R_{1}^{i(n)}, R_{2}^{i(n)}$ are a family of quasi-nonexpansive multi-valued mappings on $H_{1}, H_{2}$, respectively.

The proof of the following lemma is similar to Lemma 1.5, and we omit its proof.

Lemma 3.2 Let $T=I-\gamma G^{*} G$, where $0<\gamma<\lambda=2 / \rho\left(G^{*} G\right)$. Then we have $\operatorname{Fix}(T)=$ $\{(x, y) \in H, A x=B y\}, \operatorname{Fix}\left(P_{\cap S_{j}} T\right)=\operatorname{Fix}\left(P_{\cap S_{j}}\right) \cap \operatorname{Fix}(T)=\bar{\Gamma}$ and $\bigcap \operatorname{Fix}\left(P_{S_{j}} T\right)=\bigcap\left[\operatorname{Fix}\left(P_{S_{j}}\right) \cap\right.$ $\operatorname{Fix}(T)]=\bar{\Gamma}$.

To prove its convergence we also need the following lemma.

Lemma 3.3 Any sequence $\left\{w_{n}\right\}$ generated by Algorithm (3.1) is the Féjer-monotone with respect to $\bar{\Gamma}$, namely for every $w \in \bar{\Gamma}$,

$$
\left\|w_{n+1}-w\right\| \leq\left\|w_{n}-w\right\|, \quad \forall n \geq 1
$$

provided that $\alpha_{n}>0$ is a sequence in $(0,1)$ and $0<\gamma<\lambda=2 / \rho\left(G^{*} G\right)$.

Proof Let $u_{n}=\left(I-\gamma G^{*} G\right) w_{n}$ and taking $w \in \bar{\Gamma}$, by Lemma 3.2, $w \in \operatorname{Fix}\left(P_{S_{j}}\right) \cap \operatorname{Fix}(I-$ $\left.\gamma G^{*} G\right), \forall N \geq i \geq 1, G w=0$ and we have

$$
\begin{aligned}
\left\|w_{n+1}-w\right\|^{2} & =\left\|\alpha_{n} u_{n}+\left(1-\alpha_{n}\right) v_{n}-w\right\|^{2} \\
& \leq \alpha_{n}\left\|u_{n}-w\right\|^{2}+\left(1-\alpha_{n}\right)\left\|v_{n}-w\right\|^{2}-\alpha_{n}\left(1-\alpha_{n}\right)\left\|u_{n}-v_{n}\right\|^{2} \\
& \leq \alpha_{n}\left\|u_{n}-w\right\|^{2}+\left(1-\alpha_{n}\right) \tilde{H}\left(R_{i(n)} u_{n}-R_{i(n)} w\right)^{2}-\alpha_{n}\left(1-\alpha_{n}\right)\left\|u_{n}-v_{n}\right\|^{2} \\
& \leq \alpha_{n}\left\|u_{n}-w\right\|^{2}+\left(1-\alpha_{n}\right)\left\|u_{n}-w\right\|^{2}-\alpha_{n}\left(1-\alpha_{n}\right)\left\|u_{n}-v_{n}\right\|^{2} \\
& =\left\|u_{n}-w\right\|^{2}-\alpha_{n}\left(1-\alpha_{n}\right)\left\|u_{n}-v_{n}\right\|^{2} .
\end{aligned}
$$

On the other hand, in the same way as in the proof of Lemma 2.2, we have

$$
\left\|u_{n}-w\right\|^{2} \leq\left\|w_{n}-w\right\|^{2}-\gamma(2-\lambda \gamma)\left\|G w_{n}\right\|^{2}
$$

Hence, we have

$$
\left\|w_{n+1}-w\right\|^{2} \leq\left\|w_{n}-w\right\|^{2}-\alpha_{n}\left(1-\alpha_{n}\right)\left\|u_{n}-v_{n}\right\|^{2}-\gamma(2-\lambda \gamma)\left\|G w_{n}\right\|^{2} .
$$

It follows that $\left\|w_{n+1}-w\right\| \leq\left\|w_{n}-w\right\|, \forall w \in \bar{\Gamma}, n \geq 1$.

Theorem 3.4 If $0<\liminf _{n \rightarrow \infty} \alpha_{n} \leq \limsup _{n \rightarrow \infty} \alpha_{n}<1$, then the sequence $\left\{w_{n}\right\}$ generated by Algorithm (3.1) converges weakly to a solution of MSSEP (1.4). In addition, if there exists $1 \leq j \leq N$ such that $R_{1}^{j}, R_{2}^{j}$ are semi-compact, then $\left\{w_{n}\right\}$ converges strongly to a solution of $\operatorname{MSSEP}(1.4)$. 
Proof From (3.2), and the fact that $0<\liminf _{n \rightarrow \infty} \alpha_{n} \leq \limsup _{n \rightarrow \infty} \alpha_{n}<1$ and $0<\gamma<\lambda=$ $2 / \rho\left(G^{*} G\right)$, we obtain

$$
\sum_{n=0}^{\infty}\left\|u_{n}-v_{n}\right\|^{2}<\infty \text { and } \sum_{n=0}^{\infty}\left\|G w_{n}\right\|^{2}<\infty
$$

Therefore,

$$
\lim _{n \rightarrow \infty}\left\|u_{n}-v_{n}\right\|=0 \text { and } \lim _{n \rightarrow \infty}\left\|G w_{n}\right\|=0
$$

Since $v_{n} \in R_{i(n)} u_{n}$, we get $d\left(u_{n}, R_{i(n)} u_{n}\right) \leq\left\|u_{n}-v_{n}\right\| \rightarrow 0$.

It follows from the Féjer-monotonicity of $\left\{w_{n}\right\}$ that the sequence is bounded. Let $\tilde{w}$ be a weak-cluster point of $\left\{w_{n}\right\}$. Take a subsequence $\left\{w_{n_{k}}\right\}$ of $\left\{w_{n}\right\}$ such that $w_{n_{k}}$ converges weakly to $\tilde{w}$. Then, by Lemma 1.3 , we obtain $G \tilde{w}=0$, and it follows that $\tilde{w} \in \operatorname{Fix}\left(I-\gamma G^{*} G\right)$.

Now, by setting $u_{n}=\left(I-\gamma G^{*} G\right) w_{n}$, it follows that $u_{n_{k}}$ converges weakly to $\tilde{w}$.

Since

$$
\begin{aligned}
\left\|w_{n+1}-w_{n}\right\|^{2} & =\left\|\alpha_{n} u_{n}+\left(1-\alpha_{n}\right) v_{n}-w_{n}\right\|^{2} \\
& =\left\|\left(1-\alpha_{n}\right)\left(v_{n}-u_{n}\right)+u_{n}-w_{n}\right\|^{2} \\
& \leq 2\left(1-\alpha_{n}\right)^{2}\left\|\left(v_{n}-u_{n}\right)\right\|^{2}+2\left\|\gamma G^{*} G w_{n}\right\|^{2} \\
& =2\left(1-\alpha_{n}\right)^{2}\left\|\left(v_{n}-u_{n}\right)\right\|^{2}+2 \gamma^{2}\left\langle G w_{n}, G G^{*} G w_{n}\right\rangle \\
& \leq 2\left(1-\alpha_{n}\right)^{2}\left\|\left(v_{n}-u_{n}\right)\right\|^{2}+2 \gamma^{2} \lambda\left\|G w_{n}\right\|^{2},
\end{aligned}
$$

we have

$$
\sum_{n=0}^{\infty}\left\|w_{n+1}-w_{n}\right\|^{2}<\infty
$$

On the other hand,

$$
\begin{aligned}
\left\|u_{n+1}-u_{n}\right\|^{2} & =\left\|w_{n+1}-w_{n}+\gamma G^{*} G\left(w_{n+1}-w_{n}\right)\right\|^{2} \\
& \leq 2\left(\left\|w_{n+1}-w_{n}\right\|^{2}+\left\|\gamma G^{*} G\left(w_{n+1}-w_{n}\right)\right\|^{2}\right) \\
& \leq 2\left(\left\|w_{n+1}-w_{n}\right\|^{2}+\gamma^{2} \lambda\left\|\left(w_{n+1}-w_{n}\right)\right\|^{2}\right),
\end{aligned}
$$

that is,

$$
\sum_{n=0}^{\infty}\left\|u_{n+1}-u_{n}\right\|^{2}<\infty
$$

Thus, $\lim _{n \rightarrow \infty}\left\|u_{n+1}-u_{n}\right\|=0$ and $\lim _{n \rightarrow \infty}\left\|u_{n+j}-u_{n}\right\|=0$ for all $j=1,2, \ldots, N$.

It follows that, for any $j=1,2, \ldots, N$,

$$
\begin{gathered}
d\left(u_{n}, R_{i(n+j)} u_{n}\right) \leq\left\|u_{n}-u_{n+j}\right\|+d\left(u_{n+j}, R_{i(n+j)} u_{n+j}\right) \\
+\tilde{H}\left(R_{i(n+j)} u_{n+j}, R_{i(n+j)} u_{n}\right)
\end{gathered}
$$




$$
\leq 2\left\|u_{n}-u_{n+j}\right\|+d\left(u_{n+j}, R_{i(n+j)} u_{n+j}\right)
$$

$\rightarrow 0$.

Hence, $\lim _{n \rightarrow \infty} d\left(u_{n}, R_{j} u_{n}\right)=0$ for all $j=1,2, \ldots, N$. Since $R_{1}^{j}, R_{2}^{j}$ are demi-closed at the origin, it is easy to check that $R_{j}$ is demi-closed at the origin, and it follows that $\tilde{w} \in$ $\bigcap_{j=1}^{N} \operatorname{Fix} R_{j}=C \times Q$, i.e., $P_{S}(\tilde{w})=\tilde{w}$. That is to say $\tilde{w} \in \operatorname{Fix}\left(P_{S}\right)$. Hence $\tilde{w} \in \operatorname{Fix}\left(P_{S}\right) \cap \operatorname{Fix}(I-$ $\left.\gamma G^{*} G\right)$. By Lemma 3.2, we get that $\tilde{w}$ is a solution of the MSSEP (1.4).

The weak convergence of the whole sequence $\left\{w_{n}\right\}$ holds true since all conditions of the well-known Opial lemma (Lemma 1.4) are fulfilled with $S=\bar{\Gamma}$.

Moreover, if $R_{1}^{j}, R_{2}^{j}$ are semi-compact, it is easy to prove that $R_{j}$ is semi-compact, since $d\left(u_{n}, R_{j} u_{n}\right) \rightarrow 0$, we find that there exists a subsequence of $\left\{u_{n_{i}}\right\} \subseteq\left\{u_{n}\right\}$ such that $u_{n_{i}}$ converges strongly to $w^{*}$. Since $u_{n_{v}}$ converges weakly to $\tilde{w}$, we have $w^{*}=\tilde{w}$ and so $u_{n_{i}}$ converges strongly to $\tilde{w} \in \Gamma$. From the Féjer-monotonicity of $\left\{w_{n}\right\}$ and $\left\|w_{n+1}-u_{n}\right\|=$ $\left(1-\alpha_{n}\right)\left\|u_{n}-v_{n}\right\| \rightarrow 0$, we can see that $\left\|w_{n}-\tilde{w}\right\| \rightarrow 0$, i.e., $\left\{w_{n}\right\}$ converges strongly to a solution of the MSSEP (1.4).

\section{Competing interests}

The authors declare that they have no competing interests.

\section{Authors' contributions}

All authors contributed equally to the writing of this paper. All authors read and approved the final manuscript.

\section{Author details}

${ }^{1}$ Tianjin Vocational Institute, Tianjin, 300410, P.R. China. ${ }^{2}$ Department of Mathematics, Tianjin Polytechnic University, Tianjin, 300387, P.R. China.

\section{Acknowledgements}

This research was supported by NSFC Grants No. 11071279; No. 11226125; No. 11301379

Received: 11 September 2014 Accepted: 15 October 2014 Published: 30 Oct 2014

\section{References}

1. Censor, Y, Elfving, T: A multiprojection algorithm using Bregman projections in a product space. Numer. Algorithms 8 , 221-239 (1994)

2. Censor, Y, Elfving, T, Kopf, N, Bortfeld, T: The multiple-sets split feasibility problem and its applications for inverse problems. Inverse Probl. 21, 2071-2084 (2005)

3. Byrne, C: Iterative oblique projection onto convex sets and the split feasibility problem. Inverse Probl. 18(2), 441-453 (2002)

4. Byrne, $\mathrm{C}$ : A unified treatment of some iterative algorithms in signal processing and image reconstruction. Inverse Probl. 20(1), 103-120 (2004)

5. Qu, B, Xiu, N: A note on the CQ algorithm for the split feasibility problem. Inverse Probl. 21(5), 1655-1665 (2005)

6. Xu, HK: A variable Krasnoselskii-Mann algorithm and the multiple-set split feasibility problem. Inverse Probl. 22(6), 2021-2034 (2006)

7. Yang, Q: The relaxed CQ algorithm solving the split feasibility problem. Inverse Probl. 20(4), 1261-1266 (2004)

8. Yang, Q, Zhao, J: Generalized KM theorems and their applications. Inverse Probl. 22(3), 833-844 (2006)

9. Moudafi, A: A relaxed alternating CQ-algorithms for convex feasibility problems. Nonlinear Anal., Theory Methods Appl. 79, 117-121 (2013)

10. Shi, LY, Chen, RD, Wu, YJ: Strong convergence of iterative algorithms for the split equality problem. J. Inequal. Appl. (submitted)

11. Geobel, K, Kirk, WA: Topics in Metric Fixed Point Theory. Cambridge Studies in Advanced Mathematics, vol. 28 Cambridge University Press, Cambridge (1990)

12. Geobel, K, Reich, S: Uniform Convexity, Nonexpansive Mappings, and Hyperbolic Geometry. Dekker, New York (1984)

13. Schöpfer, F, Schuster, T, Louis, AK: An iterative regularization method for the solution of the split feasibility problem in Banach spaces. Inverse Probl. 24, 055008 (2008) 